\title{
Convergence of the Key Algorithm of Traffic-Flow Analysis
}

\author{
Gábor Lencse ${ }^{1}$ and László Muka² \\ ${ }^{1}$ Department of Telecommunications, Széchenyi István University, Győr, Hungary \\ 2 Elassys Consulting Limited, Budapest, Hungary
}

\begin{abstract}
The traffic-flow analysis (TFA) [1] is a novel method for the performance estimation of communication systems. TFA is a combination of simulation and numerical methods. In the first step, TFA distributes the traffic in units of properly chosen size using the actual routing algorithm of the network. In the second step, TFA adjusts the time distribution of the traffic according to the finite capacities of the network. The convergence of the algorithm used in the second step of TFA is proven in this paper. The speed of convergence is also examined.
\end{abstract}

Keywords: convergence, traffic-flow analysis, communication networks, performance analysis, traffic modeling, discrete-event simulation.

\section{Introduction}

The traffic-flow analysis (TFA) is a simulationlike method for the fast performance estimation of communication systems. The method can be used with any traffic model that satisfies the criteria published in [1].

Unlike discrete-event simulation, TFA does not model the travel of each packet through the network individually. It uses statistics to model the network load of applications. The method distributes traffic (the statistics) in the network first, and calculates the specific traffic conditions for each line and switching node later. This calculation requires an algorithm for the appropriate addition of the statistics considering also the finite capacities of each line and node in the network. The algorithm depends on the selected traffic model (the type of statistics). Using the throughput distribution as the traffic model, a two step algorithm was proposed in the original paper. In the first step, convolution is used to sum up all the throughput distribution statistics that were carried through a given network element (line or node). However, this summation may result in statistics that would contradict with the finite capacity of the given element. (E.g.: with a non-zero probability, higher throughput than the line capacity could occur.) For this reason, in the second step, an iterative method is applied to correct the statistics according to the finite capacity of that element. This method seemed to work fine, but its convergence was not examined in [1]. The recent paper presents a formal proof for the convergence of the method. Also, the convergence speed and stopping criteria of the algorithm are also determined so that the method can be used in a network expert system like ImiNet [2].

The paper is organized as follows: first, the problem is formalized; second, by means of a series of theorems, the convergence of the questioned algorithm is proven; finally the convergence speed is examined.

\section{Formalization of the Problem}

Parallel reading of Section 3.3 of the original paper proposing TFA [1] may help the reader to understand the role of the algorithm, but it is not necessary.

Let us consider the following block diagram.

The task of the server is to transfer the bitstream of the traffic generator through the transmission line of capacity $\boldsymbol{K}$ bits per second. We shall formalize the problem as follows. 


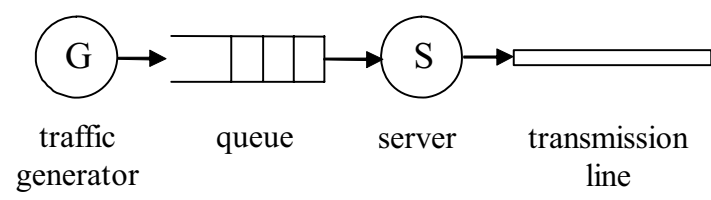

Fig. 1. The elements of the network.

Let the random variable $\boldsymbol{\xi}$ denote the length of the bitstream produced for a period of $T$ by generator $\boldsymbol{G}$. Let $\boldsymbol{m}$ denote the expected value of $\xi$ : $\mathbb{E}(\xi)=m$, and let $\boldsymbol{\sigma}$ denote the standard deviation of $\xi: D(\xi)=\sigma$. This bitstream is to be transferred through a line that can transfer $K$ bits in a $T$ time interval. Assuming that $\xi_{1}, \xi_{2}, \ldots \xi_{n}$ are independent and follow the same distribution during the consecutive $T_{1}, T_{2}, \ldots T_{n}$ time intervals (where $T_{1}=T_{2}=\ldots=T_{n}=T$ ), let $\boldsymbol{p}_{\boldsymbol{k}}$ denote the probability of the event that generator $G$ produces a bitstream of length $k$ in $T$ time: $\mathbb{P}(\xi=k)=p_{k}$. (It was denoted by $p_{T}[k]$ in [1].) Of course, $\xi$ is a non-negative discrete random variable, which can only take natural numbers as its values: $\xi \in \mathbb{N}$.

Let $\zeta^{(n)}$ denote the number of bits to be transferred in $T_{n}$ (the $n$-th time slot) through a line of capacity $K$ bits per second. $\mathbb{P}\left(\zeta^{(n)}=k\right)=p_{k}^{(n)}$. These bits may be newly arrived ones, or they may have arrived earlier and are still waiting in the queue.

The following recursive formula gives the probability of the event that the number of bits to be transferred in the $n$-th time-slot is exactly $k$ :

$$
\begin{gathered}
p_{k}^{(n)}=p_{k} \sum_{l=0}^{K} p_{l}^{(n-1)}+\sum_{l=1}^{k} p_{l+K}^{(n-1)} p_{k-l} \quad \forall k, \\
\text { where } p_{k}^{(0)}= \begin{cases}1 & \text { if } k=0 \\
0 & \text { if } k>0\end{cases}
\end{gathered}
$$

The first member of the formula expresses that only the newly arrived bits are to be transferred in the $n$-th time-slot, if the number of bits to be transferred was not greater than the $K$ capacity threshold in the $(n-1)$-th time slot (because they were all transferred and none remained waiting). The second member expresses that $l$ bits remained waiting from the previous timeslot and $k-l$ is the number of the newly arrived bits. The question of convergence is formalized as follows: $\lim _{n \rightarrow \infty} p_{k}^{(n)}=$ ?, and does a $p_{k}^{*}$ limit distribution exist?

Let $\boldsymbol{\eta}^{(n)}$ denote the number of bits transferred in the $n$-th time-slot:

$$
\eta^{(n)}= \begin{cases}\zeta^{(n)}, & \text { if } \zeta^{(n)} \leq K \\ K, & \text { if } \zeta^{(n)}>K\end{cases}
$$

\section{Proof of Convergence}

Theorem 1. Let $\xi_{1}, \xi_{2}, \ldots \xi_{n}, \ldots$ be independent random variables following the same distribution: $\mathbb{E}(\xi)=m, \mathbb{D}(\xi)=\sigma, m<K$.

$$
\lim _{n \rightarrow \infty} \mathbb{P}\left(\xi_{1}+\xi_{2}+\ldots+\xi_{n}>n K\right)=0
$$

That is, the probability of the event that the length of the bitstream produced by the generator $G$ within a period of $n T$ exceeds the line capacity, converges to 0 if $n \rightarrow \infty$.

Proof: By the central limit distribution theorem:

$$
\lim _{n \rightarrow \infty} \mathbb{P}\left(\frac{\xi_{1}+\xi_{2}+\ldots+\xi_{n}-n m}{\sigma \sqrt{n}}<x\right)=\Phi(x)
$$

where $\Phi(x)$ is the cumulative density function of the standard normal distribution:

$$
\Phi(x)=\frac{1}{\sqrt{2 \pi}} \int_{t=-\infty}^{x} e^{-\frac{t^{2}}{2}} d t
$$

$$
\begin{aligned}
& \mathbb{P}\left(\xi_{1}+\xi_{2}+\ldots+\xi_{n}>n K\right) \\
& =\mathbb{P}\left(\frac{\xi_{1}+\xi_{2}+\ldots+\xi_{n}-n m}{\sigma \sqrt{n}}>\frac{n K-n m}{\sigma \sqrt{n}}\right) \\
& =\mathbb{P}\left(\frac{\xi_{1}+\xi_{2}+\ldots+\xi_{n}-n m}{\sigma \sqrt{n}}>\frac{\sqrt{n}}{\sigma}(K-m)\right)
\end{aligned}
$$

Let $N>0$ be an arbitrary natural number. For all $N$, there exists an $n_{0}$ so that if $n>n_{0}$ then $\frac{\sqrt{n}}{\sigma}(K-m)>N$. That is, if $n>n_{0}$ then:

$$
\begin{aligned}
& \mathbb{P}\left(\frac{\xi_{1}+\xi_{2}+\ldots+\xi_{n}-n m}{\sigma \sqrt{n}}>\frac{\sqrt{n}}{\sigma}(K-m)\right) \\
& \leq \mathbb{P}\left(\frac{\xi_{1}+\xi_{2}+\ldots+\xi_{n}-n m}{\sigma \sqrt{n}}>N\right)
\end{aligned}
$$


Now, let us consider the following inequality:

$$
\begin{aligned}
& \lim _{n \rightarrow \infty} \mathbb{P}\left(\xi_{1}+\xi_{2}+\ldots+\xi_{n}>n K\right) \\
& =\lim _{n \rightarrow \infty} \mathbb{P}\left(\frac{\xi_{1}+\xi_{2}+\ldots+\xi_{n}-n m}{\sigma \sqrt{n}}\right. \\
& \left.>\frac{\sqrt{n}}{\sigma}(K-m)\right) \\
& \leq \lim _{n \rightarrow \infty} \mathbb{P}\left(\frac{\xi_{1}+\xi_{2}+\ldots+\xi_{n}-n m}{\sigma \sqrt{n}}>N\right) \\
& =1-\Phi(N) .
\end{aligned}
$$

Now, if $N \rightarrow \infty$ then $\Phi(N) \rightarrow 1$, that is: $\lim _{N \rightarrow \infty}(1-\Phi(N))=0$.

Theorem 2. Using the conditions of Theorem 1 , the probability of the event that there are waiting bits at the end of all the $T_{1}, T_{2}, \ldots, T_{n}$, intervals is likely to be 0 , if $n \rightarrow \infty$. This can be expressed as follows:

$$
\begin{aligned}
\lim _{n \rightarrow \infty} \mathbb{P}\left(\left(\xi_{1}>K\right) \wedge\left(\xi_{1}+\xi_{2}>2 K\right) \wedge \ldots\right. \\
\left.\ldots \wedge\left(\xi_{1}+\xi_{2}+\ldots+\xi_{n}>n K\right)\right)=0
\end{aligned}
$$

Proof: The event above is a subset of the event that: $\xi_{1}+\xi_{2}+\ldots+\xi_{n}>n K$.

Therefore, it is true, that:

$$
\begin{aligned}
& \mathbb{P}\left(\left(\xi_{1}>K\right) \wedge\left(\xi_{1}+\xi_{2}>2 K\right) \wedge \ldots\right. \\
& \left.\quad \ldots \wedge\left(\xi_{1}+\xi_{2}+\ldots+\xi_{n}>n K\right)\right) \\
& \leq \mathbb{P}\left(\xi_{1}+\xi_{2}+\ldots+\xi_{n}>n K\right) \\
& \mathbb{P}\left(\xi_{1}+\xi_{2}+\ldots+\xi_{n}>n K\right) \rightarrow 0 \text { if } n \rightarrow \infty \\
& \text { (see Theorem } 1) .
\end{aligned}
$$

So we have shown that:

$$
\begin{aligned}
& \lim _{n \rightarrow \infty} \mathbb{P}\left(\left(\xi_{1}>K\right) \wedge\left(\xi_{1}+\xi_{2}>2 K\right) \wedge \ldots\right. \\
& \left.\quad \ldots \wedge\left(\xi_{1}+\xi_{2}+\ldots+\xi_{n}>n K\right)\right) \\
& \leq \lim _{n \rightarrow \infty} \mathbb{P}\left(\xi_{1}+\xi_{2}+\ldots+\xi_{n}>n K\right)=0 .
\end{aligned}
$$

Corollary. If $m<K$, then, at least at the end of one of the intervals $T_{1}, T_{2}, \ldots, T_{n}$, if $n \rightarrow \infty$, there will be no waiting bits with the probability of 1 . When this event happens, we can consider it as if the process would start again from the beginning.

Definition 1. Let $\xi_{1}$ and $\xi_{2}$ be random variables. We say that $\xi_{1}$ is greater than $\xi_{2}$ by distribution, if $\forall x \in \mathbb{R}$ it is true that: $\mathbb{P}\left(\xi_{1} \geq\right.$ $x) \geq \mathbb{P}\left(\xi_{2} \geq x\right)$ and $\exists$ at least one $x_{0}$ that $\mathbb{P}\left(\xi_{1} \geq x_{0}\right)>\mathbb{P}\left(\xi_{2} \geq x_{0}\right)$. We shall denote this relation with the traditional 'greater than' sign between the 2 random variables: $\xi_{1}>\xi_{2}$.

Let us consider some features of this relation.

Theorem 3. Let $F_{1}(x)$ and $F_{2}(x)$ denote the cumulative density function of random variables $\xi_{1}$ and $\xi_{2}$, respectively.

$$
\begin{gathered}
\xi_{1}>\xi_{2} \Leftrightarrow F_{1}(x) \leq F_{2}(x) \text { and there } \\
\text { is an } x_{0}, \text { where } F_{1}\left(x_{0}\right)<F_{2}\left(x_{0}\right)
\end{gathered}
$$

Proof is provided in two parts. The proof of the $\Rightarrow$ part: As $F_{i}(x)=\mathbb{P}\left(\xi_{i}<x\right)=1-\mathbb{P}\left(\xi_{i} \geq x\right)$ $(i=1,2)$ and from definition 1 it comes that:

$$
\begin{aligned}
\mathbb{P}\left(\xi_{1} \geq x\right) & \geq \mathbb{P}\left(\xi_{2} \geq x\right) \\
1-F_{1}(x) & \geq 1-F_{2}(x) \\
F_{2}(x) & \geq F_{1}(x)
\end{aligned}
$$

And there is an $x_{0}$, where from $\mathbb{P}\left(\xi_{1} \geq x_{0}\right)>$ $\mathbb{P}\left(\xi_{2} \geq x_{0}\right)$ comes that: $F_{2}\left(x_{0}\right)>F_{1}\left(x_{0}\right)$.

The proof of the $\Leftarrow$ part is achieved by following the steps of the proof of the $\Rightarrow$ part in reverse direction.

Before further investigations, we introduce the distribution vectors.

Definition 2. $\xi$ is a non-negative discrete random variable which can take natural numbers as its values. Let us use the following notations: $p_{0}=\mathbb{P}(\xi=0), p_{1}=\mathbb{P}(\xi=1), \ldots p_{n}=$ $\mathbb{P}(\xi=n)$. The $p\left(p_{0}, p_{1}, \ldots p_{n}, \ldots\right)$ distribution vector is a finite or infinite series, which satisfies

$$
\sum_{k=0}^{\infty} p_{k}=1 \text { and } p_{k} \geq 0 \quad \forall k \in N .
$$

To all $\underline{q}\left(q_{0}, q_{1}, \ldots q_{n}, \ldots\right)$ series satisfying

$$
\sum_{k=0}^{\infty} q_{k}=1 \text { and } q_{k} \geq 0 \quad \forall k \in N
$$

we can order a distribution so that $q$ is the distribution vector of that distribution. 
Theorem 4. Let $\underline{p}\left(p_{0}, p_{1}, \ldots p_{n}, \ldots\right)$ and $\underline{q}\left(q_{0}, q_{1}, \ldots q_{n}, \ldots\right)$ be two distribution vectors.

$$
\begin{gathered}
\underline{p}>\underline{q} \Leftrightarrow\left(p_{0} \leq q_{0}\right) \wedge\left(p_{0}+p_{1} \leq q_{0}+q_{1}\right) \wedge \ldots \\
\wedge\left(\forall k \in N: \sum_{l=0}^{k} p_{l} \leq \sum_{l=0}^{k} q_{l}\right) \wedge\left(\exists k_{0} \in N:\right. \\
\left.\sum_{l=0}^{k_{0}} p_{l}<\sum_{l=0}^{k_{0}} q_{l}\right)
\end{gathered}
$$

Proof: Trivial. Use Theorem 3 and observe the cumulative density function of $\underline{p}$ and $\underline{q}$ :

$$
\begin{gathered}
F_{\underline{p}}(x)= \begin{cases}0 & \text { if } x \leq 0 \\
p_{0} & \text { if } 0<x \leq 1 \\
p_{0}+p_{1} & \text { if } 1<x \leq 2 \\
\cdots & \text { if } k<x \leq k+1 \\
\sum_{l=0}^{k} p_{l} & \end{cases} \\
F_{\underline{q}}(x)= \begin{cases}0 & \text { if } x \leq 0 \\
q_{0} & \text { if } 0<x \leq 1 \\
q_{0}+q_{1} & \text { if } 1<x \leq 2 \\
\ldots & \text { if } k<x \leq k+1 \\
\sum_{l=0}^{k} q_{l} & \end{cases}
\end{gathered}
$$

Theorem 5. Let us consider the following distribution vector series: $\underline{p} ; \underline{p}_{1}, \underline{p}_{2}, \ldots \underline{p}_{n}$, where $p$ is the distribution vector of $\xi$ (that is: $\mathbb{P}(\xi=$ $\left.\bar{k})=p_{k}\right)$ and $\underline{p}_{1}, \underline{p}_{2}, \ldots \underline{p}_{n}$ are the distribution vectors of $\zeta^{(1)}, \zeta^{(2)}, \ldots \zeta^{(n)}$, respectively. Now recall equation (1) from chapter 1. This equation defines the components $\left(p_{1}^{(n)}, p_{2}^{(n)}, \ldots p_{k}^{(n)}\right.$, ...) of the $p_{n}$ distribution vector.

$$
\underline{p}_{n}>\underline{p}_{n-1} \quad \text { for } \quad \forall n \in N^{+}
$$

Expressed in words: we face a strictly monotonously increasing series of distributions.

Proof: First, let us show that $\underline{p_{1}}>\underline{p}$ :

$$
p_{0}^{(1)}=\left(p_{0}+p_{1}+\ldots+p_{K}\right) p_{0} \leq p_{0}
$$

(We can assume that $\mathrm{P}(\xi>\mathrm{K})>0$, otherwise there would never be waiting bits at all.)

$$
\begin{aligned}
& p_{1}^{(1)}=\left(p_{0}+p_{1}+\ldots+p_{K}\right) p_{1}+p_{K+1} p_{0} \\
& \begin{aligned}
p_{0}^{(1)}+p_{1}^{(1)}=\left(p_{0}+p_{1}+\ldots+p_{K}\right)\left(p_{0}+p_{1}\right) \\
\quad+p_{K+1} p_{0}
\end{aligned} \\
& \leq\left(p_{0}+p_{1}+\ldots+p_{K}+p_{K+1}\right)\left(p_{0}+p_{1}\right) \\
& \leq p_{0}+p_{1} \text { (O.K.) }
\end{aligned}
$$

In general:

$$
\begin{aligned}
& \sum_{k=0}^{n} p_{k}^{(1)}=\sum_{k=0}^{n}\left(p_{k} \sum_{l=0}^{K} p_{l}+\sum_{l=1}^{k} p_{l+K} p_{k-l}\right) \\
& =\left(\sum_{k=0}^{n} p_{k}\right)\left(\sum_{l=0}^{K} p_{l}\right)+\sum_{l=1}^{n} p_{l+K} \sum_{k=l}^{\infty} p_{k-l} \\
& \leq \sum_{l=0}^{K} p_{l}+\sum_{l=1}^{n} p_{l+K} \\
& =\sum_{l=0}^{n} p_{l}=\sum_{k=0}^{n} p_{k} \text { (O.K.) }
\end{aligned}
$$

If $p$ has at least 2 non-zero elements and $\mathrm{P}(\xi>$ $K)^{-}>0$, then the "=" cannot always stand. So $\underline{p}_{1}>\underline{p}$ was shown.

It can be shown in a similar way that: $\underline{p}_{n}>\underline{p}_{n-1}$. In the general step, it is shown that:

$$
\sum_{k=0}^{n} p_{k}^{(n)} \leq \sum_{k=0}^{n} p_{k}^{(n-1)}
$$

$$
\begin{aligned}
& \sum_{k=0}^{n} p_{k}^{(n)}=\sum_{k=0}^{n}\left(p_{k} \sum_{l=0}^{K} p_{l}^{(n-1)}+\sum_{l=1}^{k} p_{l+K}^{(n-1)} p_{k-l}\right) \\
& =\left(\sum_{k=0}^{n} p_{k}\right)\left(\sum_{l=0}^{K} p_{l}^{(n-1)}\right)+\sum_{l=1}^{n} p_{l+K}^{(n-1)} \sum_{k=l}^{\infty} p_{k-l} \\
& \leq \sum_{l=0}^{K} p_{l}^{(n-1)}+\sum_{l=1}^{n} p_{l+K}^{(n-1)}=\sum_{l=0}^{n} p_{l}^{(n-1)}=\sum_{k=0}^{n} p_{k}^{(n-1)}
\end{aligned}
$$

Theorem 6. Let us consider again the following distribution vector series:

$$
\begin{aligned}
\underline{p}<\underline{p}_{1}< & \underline{p}_{2}<\ldots<\underline{p}_{n} \ldots \\
& \lim _{n \rightarrow \infty} p_{k}^{(n)}=p_{k}^{*} \text { for } \forall k
\end{aligned}
$$

Proof: First, let us consider $k=0$. Then $p_{0} \geq p_{0}^{(1)} \geq p_{0}^{(2)} \geq \ldots \geq p_{0}^{(n)} \geq \ldots$ is a 
monotonously decreasing series that has a lower bound of 0 . According to Weierstrass Theorem, this series is convergent. $\lim _{n \rightarrow \infty} p_{0}^{(n)}=p_{0}^{*}$. Similarly, consider the following series:

$$
\begin{aligned}
& p_{0}+p_{1} \geq p_{0}^{(1)}+p_{1}^{(1)} \geq p_{0}^{(2)}+p_{1}^{(2)} \geq \ldots \\
& \ldots \geq p_{0}^{(n)}+p_{1}^{(n)} \geq \ldots
\end{aligned}
$$

This monotonously decreasing series has a lower bound of 0 , so it is also convergent. Let us denote its limes by $\alpha_{1}$. Let us define $p_{1}^{*}$ as follows: $p_{1}^{*}=\alpha_{1}-p_{0}^{*}$. Because of the relationship between the limes and the addition operation $\left(\right.$ " + ") it is true that: $\lim _{n \rightarrow \infty} p_{1}^{(n)}=p_{1}^{*}$

Similarly, if we have already shown that the $\sum_{l=0}^{k} p_{l}^{(n)} \quad n=1,2, \ldots$ series is convergent, then, according to Weierstrass Theorem, the $\sum_{l=0}^{k+1} p_{l}^{(n)}$ $n=1,2, \ldots$ series is convergent (being a monotonously decreasing series with a lower bound 0). Its limes is: $\alpha_{k+1}=\lim _{n \rightarrow \infty} \sum_{l=0}^{k+1} p_{l}^{(n)}$. Let $p_{k+1}^{*}=\alpha_{k+1}-\sum_{l=0}^{k} p_{l}^{*}$, then $\lim _{n \rightarrow \infty} p_{k+1}^{(n)}=$ $p_{k+1}^{*}$. (Ready)

Theorem 7. Let us consider again the following distribution vector series:

$$
\begin{aligned}
& \underline{p}<\underline{p}_{1}<\underline{p}_{2}<\ldots<\underline{p}_{n} \ldots \\
& \lim _{n \rightarrow \infty} \underline{p}_{n}=\underline{p}^{*}, \text { where } \\
& \underline{p}^{*}=\left(p_{0}^{*}, p_{1}^{*}, \ldots p_{k}^{*}, \ldots\right)
\end{aligned}
$$

Proof: Besides the results of Theorem 6, we need to show that: $\sum_{k=0}^{\infty} p_{k}^{*}=1$.

We use an induction for $n$ :

$$
\begin{aligned}
& n=0 \\
& \sum_{k=0}^{\infty} p_{k}=1 \\
& n=1 \\
& \sum_{k=0}^{\infty} p_{k}^{(1)}=\sum_{k=0}^{\infty} p_{k}=1 \\
& n \quad n+1:
\end{aligned}
$$

Using, that, $\sum_{k=0}^{\infty} p_{k}^{(n)}=1$ is true for $n$, we show it for $n+1$ :

$$
\begin{aligned}
& \sum_{k=0}^{\infty} p_{k}^{(n+1)}=\sum_{k=0}^{\infty} p_{k} \sum_{l=0}^{K} p_{l}^{(n)}+\sum_{k=0}^{\infty} \sum_{l=1}^{k} p_{l+K}^{(n)} p_{k-l} \\
& \quad=\sum_{l=0}^{K} p_{l}^{(n)} \sum_{k=0}^{\infty} p_{k}+\sum_{l=1}^{\infty} \sum_{k=l}^{\infty} p_{l+K}^{(n)} p_{k-l} \\
& =\sum_{l=0}^{K} p_{l}^{(n)}+\sum_{l=1}^{\infty} p_{l+K}^{(n)} \sum_{k=l}^{\infty} p_{k-l} \\
& =\sum_{l=0}^{K} p_{l}^{(n)}+\sum_{l=K+1}^{\infty} p_{l}^{(n)}=\sum_{l=0}^{\infty} p_{l}^{(n)}=1 .
\end{aligned}
$$

That is, $\underline{p}^{*}$ is really a distribution vector.

\section{Speed of Convergence}

\subsection{Examining the Speed of Convergence}

In the following let us use a continuous approximation instead of the discrete one!

We saw that the discrete model is convergent; therefore, the smaller time interval $(T)$ we choose, the better the two models approximate each other. We can presume that the discrete model behaves similarly.

Let us suppose the amount of information produced is $m$ bits in average in a unit of time.

The quantity of information transferred under a short $[t, t+\Delta t]$ time interval is time proportionate and is equal to $g(t) \Delta t$ in average.

Regarding our system let us suppose that for small changes there is a linear relationship between the change of information transferred and the change of the amount of waiting information.

In this case the following equation can be written:

$$
\begin{gathered}
g(t+\Delta t)-g(t)=a(m \Delta t-g(t) \Delta t), \\
\text { where } a>0
\end{gathered}
$$


From the equation we get the differential equation:

$$
g^{\prime}(t)+a g(t)=a m
$$

the solution of which is:

$$
g(t)=m-\left(m-m_{0}\right) e^{-a t},
$$

where $g(0)=m_{0}$ and $g(t)$ is an exponential function, and $\lim _{t \rightarrow \infty} g(t)=m$.

In the solution there is a constant $a$ describing the speed of convergence.

Next we determine the value of $a$.

For the quantity of information transferred in time-slot $T_{n}$ the following equation can be set:

$$
\begin{gathered}
E\left(\eta^{(n)}\right) \cong g\left(T_{n}\right) T \\
=\left(m-\left(m-m_{0}\right) e^{-a n T}\right) T
\end{gathered}
$$

From this equation $a$ can be determined:

$$
a=\frac{1}{n T} \ln \frac{\left(m-m_{0}\right) T}{m T-E\left(\eta^{(n)}\right)}
$$

\subsection{The Stopping Criterion}

The quantity of information to be transferred in time interval $T_{n}$ is:

$$
\begin{aligned}
& E\left(\zeta^{(n)}\right)=(n+1) T m-\int_{0}^{n T} g(t) d t \\
& =(n+1) T m-\int_{0}^{n T} m-\left(m-m_{0}\right) e^{-a t} d t \\
& =T m+\frac{1}{a}\left(1-e^{-a n T}\right)
\end{aligned}
$$

It means that:

$$
E\left(\zeta^{(*)}\right)=\lim _{n \rightarrow \infty} E\left(\zeta^{(n)}\right)=T m+\frac{1}{a}
$$

If we want

$$
\left|E\left(\zeta^{(n)}-E \zeta^{(*)}\right)\right|<\varepsilon
$$

to be true where $\varepsilon$ is the error of approximation, then the following inequality must also stand:

$$
n_{\varepsilon}>-\frac{1}{a T} \ln (\varepsilon a)
$$

Because the continuous model is only an approximation of the discrete model, we have to test the accuracy reached in practical problems.

When testing the results on computer, we found that the method provided a correct order of magnitude for the number of steps, but to determine $\mathrm{n}_{\varepsilon}$ a multiplier between 1,2 and 1,5 should be applied.

\section{Conclusions}

A mathematical model of the key algorithm of Traffic-Flow Analysis was set up. A formal proof for the convergence of the algorithm was presented by means of a series of theorems.

The speed of convergence of the algorithm was determined and the stopping criterion was also given.

We conclude that TFA has its solid mathematical foundations and can be used as a method for fast performance estimation of communication systems.

\section{Acknowledgements}

This paper was supported by the Hungarian Academy of Sciences under the János Bolyai research grant.

It was supported in part by CBC PHARE award No. 2002/000-317-02-20.

The authors would like to express their thanks to Péter Lukács for his valuable help in probability theory issues.

\section{References}

[1] Lencse, G., Traffic-Flow Analysis for Fast Performance Estimation of Communication Systems Journal of Computing and Information Technology, Vol. 9, No. 1, March 2001. pp. 15-27.

[2] http://www.elassys.hu 
Received: April, 2005

Accepted: May, 2005

Contact address:

Gábor Lencse

Department of Telecommunications

Széchenyi István University

Egyetem tér 1 .

H-9026 Gyốr

Hungary

e-mail: lencse@sze.hu

László Muka

Elassys Consulting limited

Bég u. 3.

H-1022 Budapest

Hungary

e-mail: muka@axelero.hu

GÁBOR LENCSE received his M.Sc. in electrical engineering and computer systems from the Technical University of Budapest in 1994 and his Ph.D. in 2000. The area of his research is (parallel) discrete-event simulation methodology. He is interested in the acceleration of the simulation of communication systems. Since 1997, he works for the Széchenyi István University in Győr. He teaches computer networks and networking protocols. Now, he is an Associate Professor. Since 1998. he does R\&D in the field of the si ulation of communication systems for the Elassys Consulting Ltd. He is a member of IEEE Hungary Section, IEEE Computer Society and IEEE Communications Society.

LÁSZLÓ MUKA graduated in electrical engineering from the Technical University of Lvov in 1976. He got his special engineering degree in digital electronics from the Technical University of Budapest in 1981, and became a university level doctor in architectures of CAD systems in 1987. Dr Muka completed an MBA at Brunel University of London in 1996. Since 1996 he has been working in the area of simulation modelling of telecommunication systems, including human subsystems. He is a regular invited lecturer in the topics of application of computer simulation for performance analysis of telecommunication systems, at the Széchenyi István University of Győr. 